\title{
O UNIVERSO ${ }^{+*}$
}

Kepler de Souza Oliveira Filho

Departamento de Astronomia - UFRGS

Porto Alegre - RS

\section{Resumo}

O Universo é finito no tempo e no espaço e as observações atuais indicam que ele está se expandindo com velocidade acelerada.

Palavras-chave: Big Bang, Radiação Cósmica do Fundo do Universo; evolução química do Universo; Einstein; Gamow.

\begin{abstract}
The Universe is finite in space and time and the present day observations indicate it is expanding at an accelerated rate.
\end{abstract}

Keywords: Big Bang; Cosmic Microwave Background Radiation; chemical evolution of the Universe; Einstein; Gamow.

\section{Introdução}

Apesar de fortes restrições interiores, o homem teve, aos poucos, de abandonar a noção de que tinha qualquer posição central no Universo e, no começo do século $\mathrm{XX}$, reconheceu que vivemos num planeta nada excepcional, a Terra, que gira em torno de uma estrela comum, o Sol, localizada quase na extremidade de uma galáxia normal, a Via Láctea.

\footnotetext{
The Universe

* Recebido: julho de 2010.

Aceito: julho de 2010.
} 
Essa galáxia faz parte de um grupo de galáxias, o Grupo Local, localizado na periferia de um grande aglomerado de galáxias.

Mesmo esse aglomerado, o aglomerado de Virgem, é pequeno em comparação aos grandes aglomerados de galáxias que podemos observar em outras partes do Universo. Nossa localização no Universo é, portanto, insignificante.

\section{O Paradoxo de Olbers: a escuridão da noite}

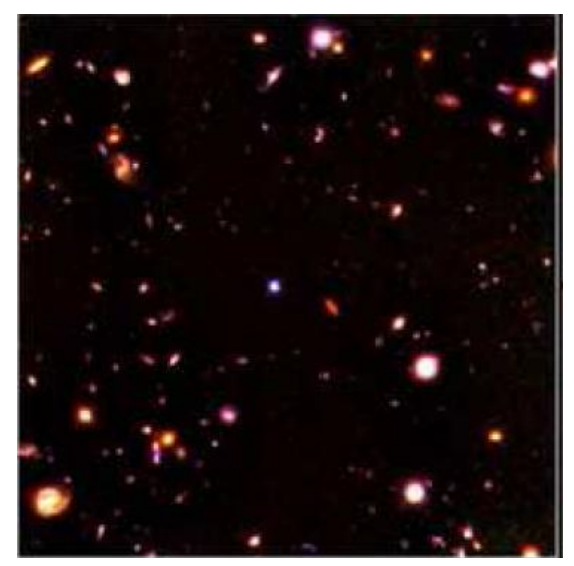

Uma das constatações mais simples que podemos fazer é que o céu é escuro à noite. É estranho que esse fato, sobre o qual ninguém, em sã consciência, colocará qualquer dúvida e que, à primeira vista, parece tão compreensível para qualquer pessoa, tenha dado tanto o que pensar durante tanto tempo.

Aparentemente, a primeira pessoa que reconheceu as implicações cosmológicas da escuridão noturna foi Johannes Kepler (1571-1630), em 1610.

Kepler rejeitava veementemente a idéia de um Universo infinito recoberto de estrelas, que, nessa época, estava ganhando vários adeptos, principalmente depois da comprovação por Galileu Galilei (1569-1642) de que a Via Látea era composta de uma miríade de estrelas e usou o fato de que o céu é escuro à noite como argumento para provar que o Universo era infinito, como que encerrado por uma parede cósmica escura.

A questão foi retomada por Edmond Halley (1656-1742) no século XVIII e pelo médico e astrônomo Heinrich Wilhelm Mattäus Olbers (1758-1840) em 1826, quando passou a ser conhecida como paradoxo de Olbers. Olbers já havia descoberta os dois asteróides (planetas menores) Palas, em 1802, e Vespa em 1807. 
O problema é o seguinte: suponha que as estrelas estejam distribuídas de maneira uniforme em um espaço infinito. Para um observador em qualquer lugar, o volume de uma esfera com centro nele aumentará com o quadrado do raio dessa esfera $\left(d V=4 \pi R^{2} d r\right)$. Portanto, à medida que ele olha mais longe, vê um número de estrelas que cresce com o quadrado da distância. Como resultado, sua linha de visada sempre interceptará uma estrela, seja lá qual for a direção em que ele olhe.

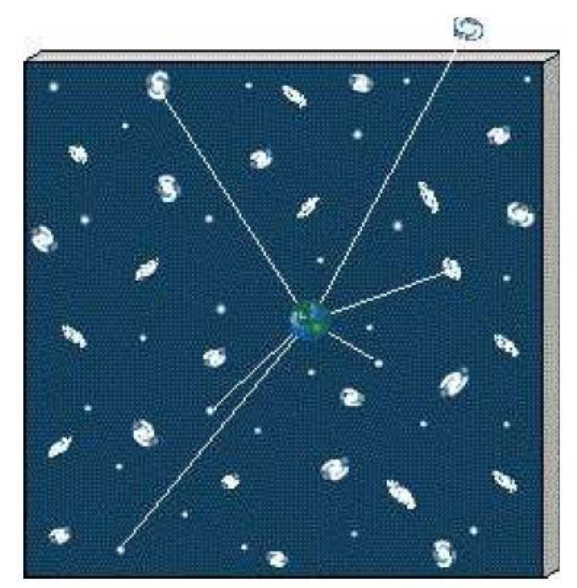

Uma analogia simples de fazer é com uma floresta de árvores. Se estamos no meio da floresta, a nosso redor vemos as árvores bem espaçadas entre si, mas quanto mais longe olhamos, mais diminui o espaçamento entre as árvores, de forma que, no limite da nossa linha de visada, as árvores estarão todas juntas e nada podemos ver além delas.

Como o brilho das estrelas cai com o quadrado da distância, enquanto o número de estrelas aumenta com o quadrado da distância, o céu, em média, deveria ser tão brilhante quanto a superfície de uma estrela média, pois estaria completamente coberto delas. Mas obviamente, não é isso que vemos e, portanto, o raciocínio está errado. Por quê?

O Universo não existiu por todo o sempre. Essa é a solução atualmente aceita para o paradoxo. Como o Universo tem uma idade finita, e a luz tem uma velocidade finita, a luz das estrelas mais distantes ainda não teve tempo de chegar até nós. Portanto, o Universo que enxergamos é limitado no espaço, por ser finito no tempo. A escuridão da noite é uma prova de que o Universo teve um início.

Usando-se a separação média entre as estrelas de um parsec, obtém-se que o céu seria tão luminoso quanto a superfície do Sol se o Universo tivesse um raio 
de $6,6 \times 10^{15}$ anos-luz. Como o Universo só tem 13,7 bilhões de anos, sua idade finita é a principal explicação ao Paradoxo de Olbers.

\section{Relatividade geral}

Em 1905, Albert Einstein (1879-1955) havia proposto a teria da relatividade especial. Essa teoria propunha que a velocidade da luz no vácuo é constante, que independente da velocidade da fonte, que a massa depende da velocidade, que há dilatação do tempo durante movimento em alta velocidade, que massa e energia são equivalentes, que nenhuma informação ou matéria pode se mover mais rápido do que a luz. A teoria é especial somente porque estava restrita ao caso em que os campos gravitacionais são pequenos, ou desprezíveis. Embora a teoria de relatividade geral, proposta por Einstein em 1916, só difira da teoria da gravitação de Isaac Newton (Newton (1643-1726) em poucas partes em um milhão na Terra, em grandes dimensões massas, como o Universo, ela resulta bastante diferente. A teoria da relatividade geral é universal no sentido de ser válida mesmo nos casos em que os campos gravitacionais não são desprezíveis. Trata-se, na verdade, da teoria da gravidade, descrevendo a gravitação como a ação das massas nas propriedades do espaço e do tempo, que afetam, por sua vez, o movimento dos corpos e outras propriedades físicas. Enquanto, na teoria de Newton, o espaço é rígido, descrito pela geometria Euclidiana [Euclides de Alexandria (c.365-300 a. C.)], na relatividade geral o espaço-tempo é distorcido pela presença da matéria que ele contém.

Um ano depois de propor a relatividade geral, em 1917, Einstein publicou seu artigo histórico sobre cosmologia, Considerações Cosmológicas sobre a Teoria da Relatividade, construindo um modelo esférico do Universo. Como as equações da Relatividade Geral não levaram diretamente a um Universo estático de raio finito, mesma dificuldade encontrada com a teoria de Newton, Einstein modificou suas equações, introduzindo a constante cosmológica, para obter um Universo estático, já que ele não tinha, naquela época, nenhuma razão para supor que o Universo estivesse se expandindo ou contraindo.

A constante cosmológica age como uma força repulsiva que previne o colapso do Universo pela atração gravitacional.

A solução de Einstein é homogênea, isto é, tem a mesma forma de qualquer ponto do espaço, e isotrópica, isto é, o modelo é o mesmo em qualquer direção. A hipótese que o Universo seja homogêneo e isotrópico é chamada de Princípio Cosmológico. 
O holandês Willen de Sitter (1872-1934) demonstrou, em 1917, que a constante cosmológica permite um Universo em expansão mesmo se ele não contiver qualquer matéria e, portanto, ela é também chamada de energia do vácuo.

As observações mostram que o Universo é homogêneo em escalas de 10 a 100 milhões de anos-luz e maiores.

Para escalas menores, podemos ver estrelas, galáxias e aglomerados de galáxias, mas, em larga escala, os elementos de volume são homogêneos.

\section{Lentes Gravitacionais}
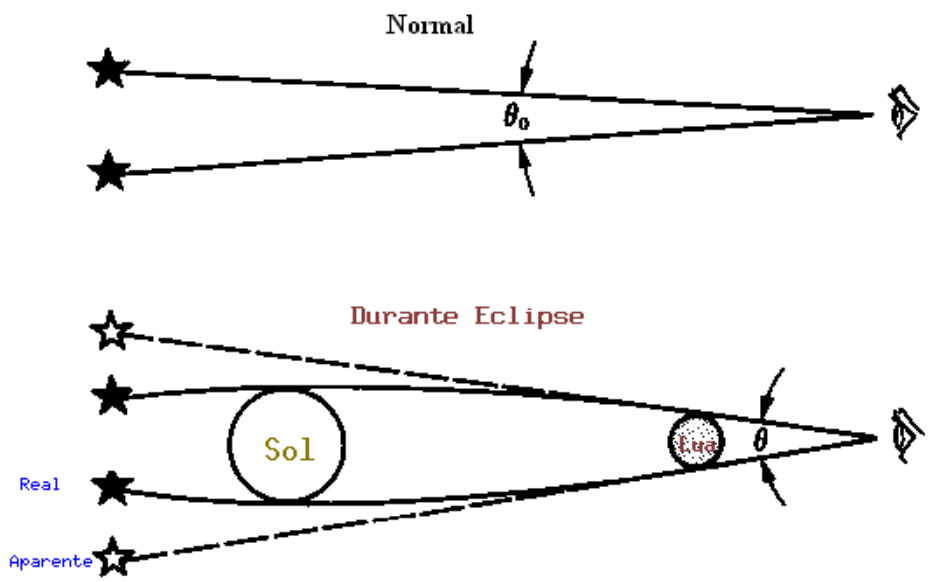

A previsão da relatividade geral de que um raio de luz é desviado ao passar por um corpo massivo foi confirmada, em 1919, por uma expedição dupla chefiada pelo astrônomo inglês Sir Arthur Stanley Eddington (1882-1944), a Sobral, no Ceará, e à ilha de Príncipe, na África, para medir a posição das estrelas durante o eclipse do Sol de 29 de maio de 1919.

A expedição ao Brasil foi coordenada pelo inglês Andrew Claude de la Cherois Crommelin (1865-1939) e retornou com 7 fotografias de boa qualidade. Medindo a distância entre as estrelas à esquerda do Sol e as estrelas à direita do Sol durante o eclipse, quando as estrelas estão visíveis pelo curto espaço de tempo do eclipse, e comparando com medidas das mesmas estrelas obtidas 2 meses depois, 
quando elas eram visíveis à noite, Eddington verificou que as estrelas pareciam mais distantes umas das outras durante o eclipse.

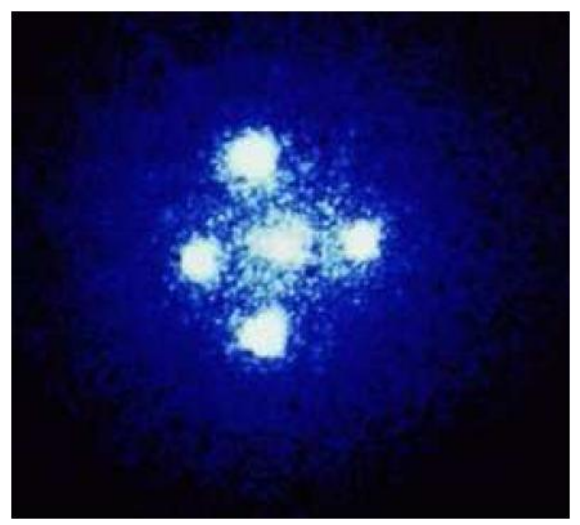

Fig. 1: Imagem do Cruz de Einstein, a lente gravitacional G2237+0305, fotografada com a Faint Object Camera, da European Space Agency, instalada no Telescópio Espacial Hubble, da NASA. A luz de um quasar distante forma quatro imagens ao passar pelo campo gravitacional de uma galáxia entre o quasar e a Terra. O quasar está a, aproximadamente, 8 bilhões de anos-luz de nós, enquanto que a galáxia está a 400 milhões de anos-luz. O número de imagens produzidas depende da distribuição de massa da galáxia e dos detalhes do alinhamento.

Isso implica que os raios de luz dessas estrelas foram desviados pelo campo gravitacional do Sol, como predito por Einstein. O desvio previsto era de

$$
\theta-\theta_{o}=\frac{1,7 \text { segundos de arco }}{\Delta} \text {, }
$$

a uma distância de $\Delta$ raios do Sol do centro do Sol. As duas explicações obtiveram 1,98 " $\pm 0,30$ " e $1,61 " \pm 0,30$ ", confirmando a teoria.

A única razão de realizar essas medidas durante um eclipse é que, durante um eclipse, podemos enxergar e medir as estrelas próximas ao disco do Sol.

Outra comprovação importante da Teoria da Relatividade Geral foi a observação do deslocamento do periélio do planeta Mercúrio, de 43" por século, já detectado pelo francês Urbain Jean Le Verrier (1811-1877), em 1859, que não 
pode ser explicado pela teoria Newtoniana, mas é perfeitamente descrito pela teoria da relatividade.

Enquanto na teoria de Newton somente a massa contribui para a gravidade, na teoria de Einstein a energia cinética do movimento dos planetas também contribui. O periélio de Vênus também se desloca, mas de 8,6 " por século, e o da Terra de 3,8" por século, ambos já medidos.

Mas a observação mais crucial, ainda, é a da medida da taxa de redução do período orbital do pulsar binário PSR 1913+16 - duas estrelas de nêutrons descoberto por Russell Alan Hulse (1950-) e Joseph Hooton Taylor Jr. (1941-) em 1974, utilizando a antena de $305 \mathrm{~m}$ de diâmetro do rádio-telescópio de Arecibo.

O período orbital é de 7,75 horas, e o período de rotação do pulsar de 59 milissegundos. A taxa de redução do período orbital, de $(76,0 \pm 0,3)$ milionésimos de segundos por ano, concorda com precisão melhor do que $1 \%$ com o cálculo de perda de energia devido à emissão de ondas gravitacionais, previstas pela teoria de Einstein ${ }^{1}$. Essa descoberta lhes valeu o prêmio Nobel de Física de 1993.

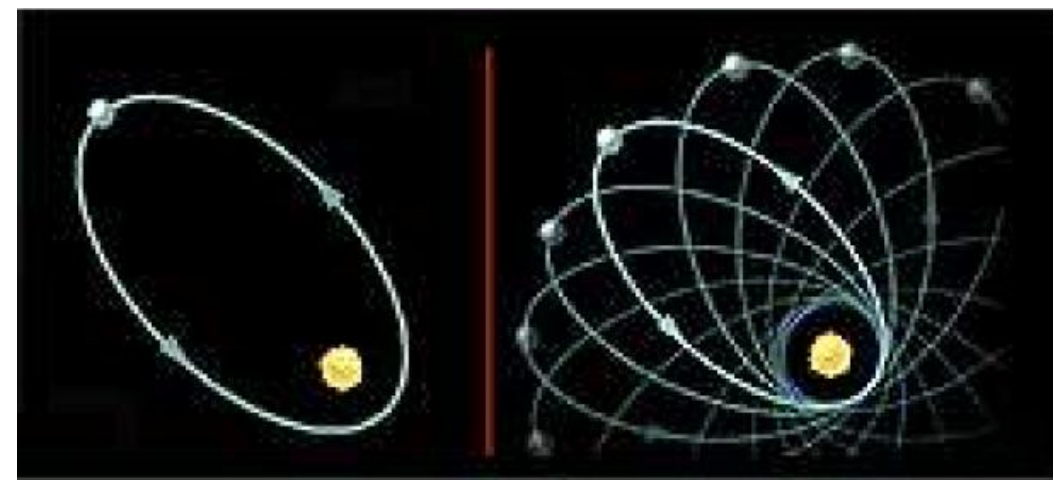

Fig. 2: Representação do deslocamento do periélio de Mercúrio com o tempo. O espaço-tempo é perturbado pela presença da massa do Sol, exatamente como predito pela Teoria da Relatividade Geral.

\footnotetext{
${ }^{1}$ A Teoria da Relatividade Geral prediz que massas aceleradas emitem ondas gravitacionais, da mesma maneira que cargas elétricas aceleradas produzem ondas eletromagnéticas. As ondas gravitacionais são perturbadas na curvatura do espaço-tempo e se propagam à velocidade da luz. Uma onda gravitacional proveniente de uma fonte intensa, como um pulsar binário próximo, altera as distâncias, mas por fatores da ordem de $10^{-21}$.
} 

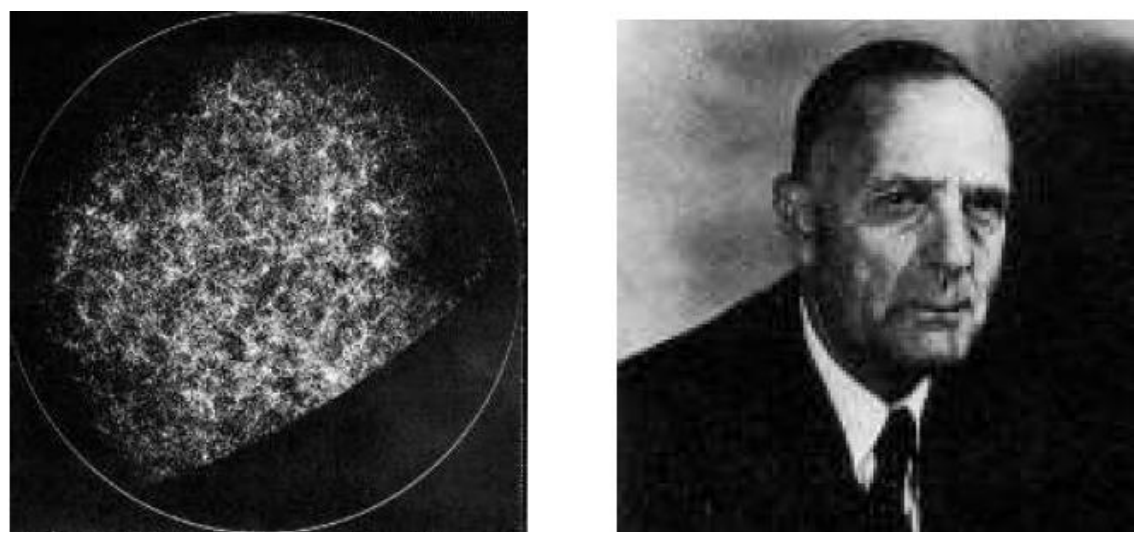

Fig. 3: Distribuição de galáxias em grande escala, e foto de Edwin Hubble.

\section{Expansão do Universo}

Em 1923, o astrônomo americano Edwin Powell Hubble (1889-1953), usando o recém-instalado telescópio de 2,5 m de diâmetro do Monte Wilson, na Califórnia, conseguiu enxergar e medir as estrelas individuais na galáxia de Andrômeda, muito mais distante do que o tamanho de nossa galáxia, demonstrando conclusivamente que nossa galáxia não é a única no Universo.

Em 1929, Hubble demonstrou, observando o deslocamento para o vermelho nas linhas espectrais das galáxias observadas por Milton La Salle Humason (1891-1972) e medindo, ele próprio, suas distâncias, que a maioria das galáxias estava se afastando de nós, com velocidades proporcionais às suas distâncias, isto é, quanto mais distante a galáxia, maior sua velocidade de afastamento.

Isso constituiu a primeira evidência para a expansão do Universo, já predita pelo russo Alexander Alexandrovitch Friedmann (1888-1925) em dois artigos publicados no Zeitschrift für Physik em 1922 e 1924, e pelo belga Georges-Henri Édouard Lemaître (1894-1966) em 1927, no Annales de la Société Scientifique de Bruxelles. 

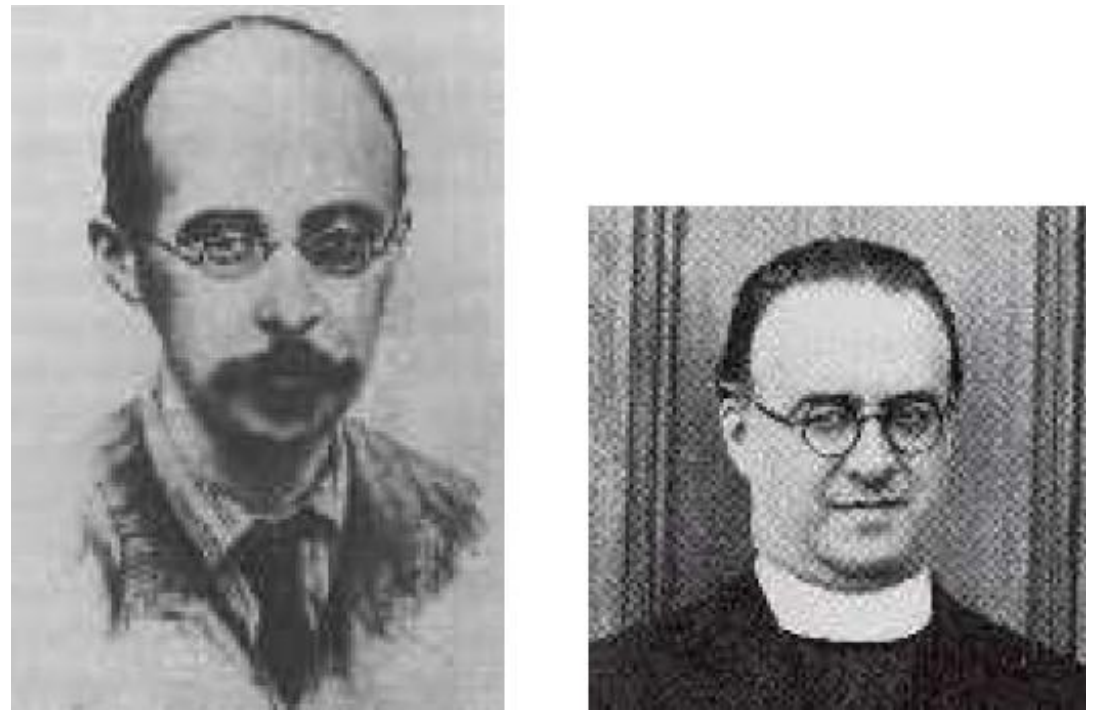

Fig. 4: Alexander Friedmann e Georges Lemaître.

Apesar da descoberta da expansão do Universo, muitos pesquisadores acreditavam na Teoria do Estado Estacionário ${ }^{2}$, isto é, que o Universo era similar em todas as direções e imutável no tempo, com produção contínua de matéria para contrabalançar a expansão observada, mantendo a densidade média constante. Essa teoria foi proposta por Sir Herman Bondi (1919-2005), Thomas Gold (1920-2004) e Sir Fred Hoyle (1915-2001).

Em 1950, Fred Hoyle sugeriu, pejorativamente, o nome "Big Bang" ou Grande Expansão, para o evento de início do Universo, quando se iniciou a expan-

${ }^{2}$ Fred Hoyle (1915-2001), Geoffrey Burbidge (1925-) e Jayant Vishnu Narlikar (1938-) propuseram, em 1993, a Teoria do Estado do Estado Quase Estacionário, em um Universo eterno e infinito, alternando expansões que duram cerca de 40 bilhões de anos, com contrações. A massa é eternamente criada em buracos brancos com massa de Plank $\sqrt{\frac{c h}{G}}=10^{19}$ bárions. A minicriação causa uma expansão do Universo, que reduz o valor médio do campo de criação, reservatório de energia negativa. Após a expansão, o valor do campo se reduz, tornando-se difícil uma nova minicriação. A gravidade, então, supera a expansão e o Universo se contrai, aumentando o campo até que nova criação ocorra. 
são. Edward P. Tryon propôs, em 1973 (Nature, 246, 396), que o Big Bang ocorreu por uma flutuação quântica do vácuo ${ }^{3}$. Já em relação ao destino do Universo, há duas possibilidades: 1) o Universo se expandirá para sempre, ou 2) a expansão parará e haver novo colapso ao estado denso (Big Crunch). O Universo colapsará novamente somente se a atração gravitacional da matéria (e energia) contida nele for grande o suficiente para parar a expansão. Como a matéria e energia escura ${ }^{4}$ do Universo pode chegar a $96 \%$ da energia total, aparentemente o Universo está se expandido com velocidade maior do que a velocidade de escape, isto é, o Universo continuará se expandindo para sempre. Podemos expressar a massa em termos da densidade, isto é, da massa por unidade de volume. A densidade crítica, que interromperia a expansão, é de 100 milésimos de trilionésimos de trilionésimos de um grama por centímetro cúbico.

$$
\rho_{\text {critica }} \cong 10^{-29} \mathrm{~g} / \mathrm{cm}^{3}=10^{-26} \mathrm{~kg} / \mathrm{m}^{3}
$$

Essa densidade crítica corresponde a 5 átomos de hidrogênio por metro cúbico, dez milhões de vezes menor do que o melhor vácuo que pode ser obtido em

\footnotetext{
${ }^{3}$ Se a energia total do Universo for nula, isto é, Universo plano na forma mais simples, então, pelo princípio da incerteza de Heisenberg $\Delta t \geq \hbar / \Delta E$ pode ser muito grande, permitindo que o Universo alcance sua idade atual. Mas por que a flutuação, que é um buraco negro por conter toda a massa do Universo em um raio muito pequeno, não colapsa? Porque a liberação de energia do calor latente da transição de fase da Teoria da Grande Unificação, separando a força gravitacional das outras no tempo de Planck, faz o Universo se expandir exponencialmente. Em 1948, os físicos holandeses Hendrik Brugt Gerhard Casimir (19092000) e Dirk Polder (1919-2001), do Philips Researh Laboratories, propuseram a existência de uma força (energia) no vácuo, devido a flutuações quânticas do vácuo. Essa força foi primeiro medida por Marcus Spaarnay, também da Philips, em 1958, mas mais precisamente em 1997, por Steve K. Lamoreaux, do Los Alamos National Laboratory, e por Umar Mohideen, da University of California em Riverside, e seu colaborador Anushree Roy.

${ }^{4}$ Aristóteles de Estagira (384-322 a.C.) propôs que a matéria na Terra era composta por quatro elementos básicos: terra, ar, fogo e água e que a matéria celeste era composta por um tipo de matéria especial, a quinta-essência, ou quintessência. Nos últimos anos, tem-se usado o termo quintessência para descrever a matéria (energia) dominante no Universo, seja ela matéria escura ou energia do vácuo (constante cosmológica).
} 
um laboratório na Terra. A matéria visível do Universo é, ainda, em média, 100 vezes menor ${ }^{5}$.

$$
\rho_{\text {observada em matéria lumin osa }} \cong 10^{-31} \mathrm{~g} / \mathrm{cm}^{3} \cong 10^{-28} \mathrm{~kg} / \mathrm{m}^{3}
$$

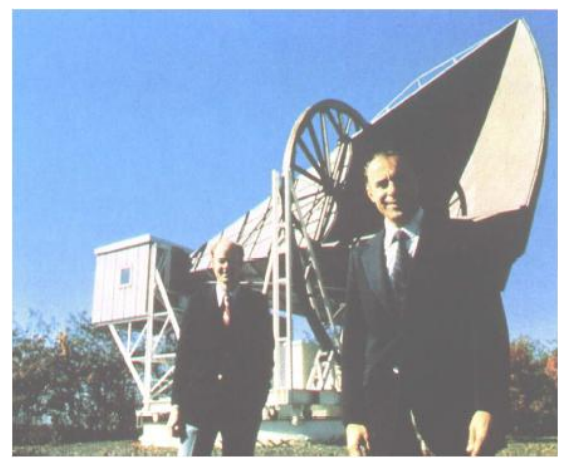

Arno Penzias e Robert Wilson com sua antena corneta de Holmdel, com a qual descobriram o excesso de energia devido à radiação cósmica do fundo do Universo, utilizando o amplificador maser de baixissimo ruido que haviam desenvolvido no Bell Labs.

Em 1964, a descoberta acidental da radiação de microondas do fundo do Universo pelos radioastrônomos Arno Allan Penzias (1933-) e Robert Woodrow Wilson (1936-), do Bell Laboratories, reforçou a teoria do Big Bang. Penzias e Wilson, que receberam o prêmio Nobel em 1978, publicaram seus resultados do excesso de emissão observado no Astrophysical Journal, em 1965 e, no mesmo

\footnotetext{
5 A densidade de matéria luminosa no Grupo Local pode ser estimada considerando que a Via Láctea e Andrômeda, as duas maiores galáxias do Grupo, têm cerca de $2 \times 10^{11} \mathrm{M} \odot$ cada, as outras 38 galáxias anãs têm massa similiar à da Grande Nuvem de Magalhães, cerca de $6 \times 10^{9} \mathrm{M} \odot$ cada, a massa total do aglomerado é $6 \times 10^{12} \mathrm{M} \odot$. O volume total do é de uma esfera de $1000 \mathrm{kpc}$ de raio, que dá $4 \times 10^{9} \mathrm{kpc}^{3}$, ou seja,

$$
\rho_{\text {luminosa }} \cong 150 \mathrm{M} \odot / \mathrm{kpc}^{3} \cong 1 \times 10^{-26} \mathrm{~kg} / \mathrm{m}^{3},
$$

superestimada porque a distância dos cúmulos de galáxias é muito maior do que a distância entre as galáxias.
} 
volume, Robert Henry Dicke (1916-1997), Philip James Edward Peebles (1935-), Peter G. Roll e David T. Wilkinson (1935-2002), que estavam construindo uma antena para procurar por essa emissão, publicaram a interpretação do excesso como a detecção da radiação remanescente do Big Bang.

A radiação do fundo do Universo é o sinal eletromagnético proveniente das regiões mais distantes do Universo (a cerca de 12 bilhões de anos-luz); ela havia sido predita, em 1948, pelos americanos Ralph Asher Alpher (1921-) e Robert Herman (1922-1997), associados a George Antonovich Gamow (1904-1968), como a radiação remanescente do estado quente em que o Universo se encontrava quando se formou (na verdade, quando ele ficou transparente, 380000 anos após o início, há 13,7 bilhões de anos). Ralph Alpher e Robert Herman publicaram a previsão da radiação do fundo do Universo, 5K, em 1948, na Nature, 162, 774.

\section{Big Bang}

A teoria do Big Bang leva em conta que, se as galáxias estão se afastando umas das outras, como observado por Edwin Hubble em 1929, no passado, elas deveriam estar cada vez próximas e, num passado remoto, 10 a 15 bilhões de anos atrás, deveriam estar todas num mesmo ponto, muito quente, uma singularidade espaço-tempo, que se expandiu no Big Bang. O Big Bang, ou Grande Expansão, criou não somente a matéria e a radiação, mas também o próprio espaço e o tempo. Esse seria o início do Universo observável.

A expansão do Universo não influi no tamanho das galáxias e cúmulos de galáxias, que são mantidos coesos pela gravidade; o espaço entre eles simplesmente aumenta, como num bolo com passas, crescendo com fermento no forno.

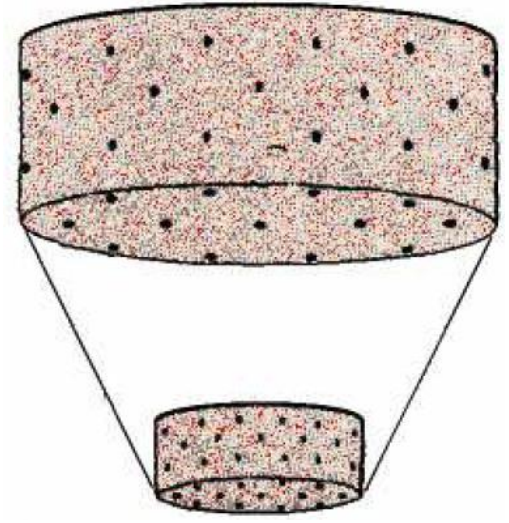


O padre, engenheiro civil e cosmólogo belga Georges-Henri Édouard Lemaitre (1894-1966) foi, provavelmente, o primeiro a propor um modelo específico para o Big Bang, em 1927. Ele imaginou que toda a matéria estivesse concentrada no que ele chamou de átomo primordial e que esse átomo se partiu em incontáveis pedaços, cada um se fragmentando cada vez mais, até formar os átomos presentes no Universo, numa enorme fissão nuclear. Sabemos que esse modelo não pode ser correto, pois não obedece às leis da relatividade e estrutura da matéria (quântica), mas ele inspirou os modelos modernos.

Independentemente de Lemaître, o matemático e meteorologista russo Alexander Alexandrovitch Friedmann (1888-1925) já tinha descoberto toda uma família de soluções das equações da Teoria da Relatividade Geral.

A família de soluções para a relatividade geral encontrada por Friedmann e Lemaître descreve um Universo em expansão, e eles são chamados os pais da Cosmologia. As soluções possíveis das equações da relatividade geral incluem expansão eterna ou com recolapso. Se a constante cosmológica é nula, os modelos se dividem em três classes. Se a densidade de matéria for alta suficiente para reverter a expansão, o Universo é fechado, como a superfície de uma esfera, mas em três dimensões, de modo que, se uma nave viajasse por um tempo extremamente longo em linha reta, voltaria ao mesmo ponto. Se a densidade for muito baixa, o baixo é aberto e continuará se expandindo para sempre. O terceiro caso, chamado de Universo plano, é o limite entre o Universo aberto e o fechado. O Universo, nesse caso, se expande para sempre, mas a velocidade das galáxias será cada vez menor, chegando a zero no infinito. Nesse caso, o Universo é euclidiano.

Qual desses modelos representa o Universo real continua como um dos cernes da cosmologia moderna, mas as observações recentes começam a testar essas hipóteses.

Em abril de 2001, o projeto Boomerang publicou nova análise dos dados de microondas por balão, com resolução de $0,3^{0}$ (comparados com $7^{0}$ do COBE) que mediram $1,8 \%$ do céu, e concluíram que a matéria bariônica só representa $3 \%$ da energia total, que a energia total está entre 0,98 e 1,03 da energia crítica e que a energia de repulsão está entre 0,52 e 0,68 da energia crítica. Com esses resultados, a idade do Universo está entre 14 e 16,2 Ganos.

O satélite WMAP (Microwave Anisotropy Probe) foi lançado em 30 de junho de 2001 e, com uma resolução de $0,21^{0}$ e uma sensibilidade de 20 micro Kelvins, fez uma medida muito mais detalhada de todo o céu, como discutiremos. A radiação do fundo do Universo mostra as condições do Universo 380 mil após o Big Bang, quando o Universo era dominado por radiação. Aproximadamente 380 mil anos depois do Big Bang, a temperatura do Universo caiu para cerca de 
$3000 \mathrm{~K}$, suficiente para que os prótons e as partículas- $\alpha$, formadas nos três primeiros minutos do Universo, começassem a capturar elétrons, e formar átomos de hidrogênio e hélio neutros. Peebles chamou essa fase de recombinação, ou fase de desacoplamento, passando para Universo dominado por matéria.

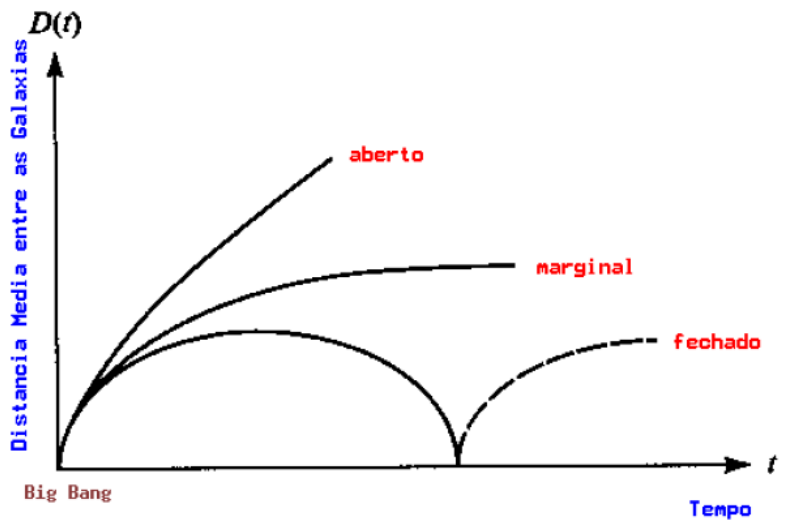

Em 1940, o físico russo-americano George Gamow, que fora estudante de Friedmann antes da morte deste aos 37 anos, sugeriu um modelo com início oposto ao de Lemaître: fusão nuclear. Ele publicou os resultados em 1948, com Ralph Alpher [e Hans Bethe (1906-2005)]. Esse modelo iniciou com partículas fundamentais, que se aglomeraram em elementos mais pesados, por fusão, após o Big Bang. Suas idéias estão corretas, exceto que as condições iniciais do Universo não eram apropriadas para fundir o carbono e elementos mais pesados, formando somente $\mathrm{H}$ e $\mathrm{He}$ em abundância significativa. Os elementos mais pesados foram produzidos, mais tarde, no interior das estrelas.

\section{A questão da matéria escura}

Outro item importante na cosmologia é a chamada matéria escura, postulada pela primeira vez por Fritz Zwicky (1898-1974) e Walter Baade (1893-1960) em 1937 (Astrophysical Journal, 86, 217).

Essa é o excesso de matéria necessário para explicar as curvas de rotação das galáxias e as velocidades observadas das galáxias em aglomerados, maiores que as explicáveis através da matéria observada, chamada matéria luminosa.

Zwicky, um astrônomo suíço trabalhando nos Estados Unidos, observando que as velocidades das galáxias em aglomerados eram muito maiores do que deve- 
riam ser, calculou que a massa do aglomerado deveria ser, pelo menos, dez vezes maior do que a massa da matéria visível no aglomerado, isto é, da massa em estrelas e gás pertencentes às galáxias.

Em 1980, Vera Cooper Rubin (1928-) determinou, pelas velocidades de rotação das galáxias, que a matéria escura também está presente em galáxias individuais (Astrophysical Journal, 238, 808).

A matéria escura tem implicações importantes nos modelos de Big Bang, como o do Universo Inflacionário. Esse modelo de Universo, proposto em 1979 por Alan Harvey Guth (1947-), do Massachussets Institute of Technology (MIT), nos Estados Unidos, e modificado em 1981 pelo russo Andrei Dmitrvitch Linde (1948-), e pelo americano Paul J. Paul Steinhardt (1952-), é consistente com algumas das formas das Teorias da Grande Unificação (GUT) das forças forte e eletrofraca, que prevêem uma quebra de simetria espontânea $10^{-37} \mathrm{~s}$ depois do Bing Bang.

Essa quebra de simetria, ou transição de fase, é causada por um falso vácuo, um estado metaestável do campo de energia que, tendo pressão negativa, faz a gravitação agir repulsivamente, expandindo o Universo um fator de $10^{75}$. Depois de $10^{-36} \mathrm{~s}$, a teoria é idêntica ao Big Bang padrão.

Outra interpretação da mesma transição de fase é que a liberação do calor latente é que faz o Universo se expandir inflacionariamente.

Quando publicada, em 1979, a transição de fase (superesfriamento) era prevista ter ocorrido em $10^{-35} \mathrm{~s}$, mas o valor moderno da energia de Higgs [Peter Ware Higgs (1929-)] é de $10^{16} \mathrm{GeV}$, correspondente a $10^{-37} \mathrm{~s}$ pelo princípio da incerteza. O bóson de Higgs é a partícula que dá massa a todas as outras partículas, no Modelo Padrão das forças nucleares.

Enquanto, no modelo inicial de Guth, nosso Universo seria composto de muitas bolhas que se expandem exponencialmente, o que é inconsistente com a uniformidade da radiação do fundo do Universo, nos novos modelos inflacionários de Linde e Steinhardt, nosso Universo é apenas uma bolha de um possível megauniverso de bolhas.

A teoria inflacionária prevê que a matéria escura não pode ser totalmente bariônica, mas é consistente com matéria escura fria, isto é, partículas com velocidade muito menor do que a velocidade de luz (neutrinos devem ter velocidade próxima à da luz). $\mathrm{O}$ modelo inflacionário prevê, ainda, que o Universo contém cem vezes mais matéria ou energia escura que a matéria que brilha nas estrelas e, portanto, que o Universo é plano.

Esse modelo explicaria as grandes paredes e buracos observados na estrutura de grande escala do Universo, e que não estão casualmente conectadas atualmente, mas o seriam antes da expansão inflacionária. Diz-se que duas regiões não 
estão casualmente conectadas se, quando a radiação foi emitida por elas, as regiões no espaço estavam mais distantes do que a distância que a luz poderia ter atravessado desde o Big Bang. Entretanto, a mesma Teoria de Grande Unificação, que prediz o Universo inflacionário, também prediz que os prótons deveriam decair em $10^{30}$ anos, o que não é observado ( $\tau_{\mathrm{obs}}>10^{33}$ anos), de modo que as teorias mais simples da GUT já foram eliminadas. Teorias de grande unificação, que permitem a quebra de simetria que formou a assimetria de matéria-antimatéria antes de $10^{-32}$ segundos, ainda são consistentes com o tempo de decaimento observado do próton.

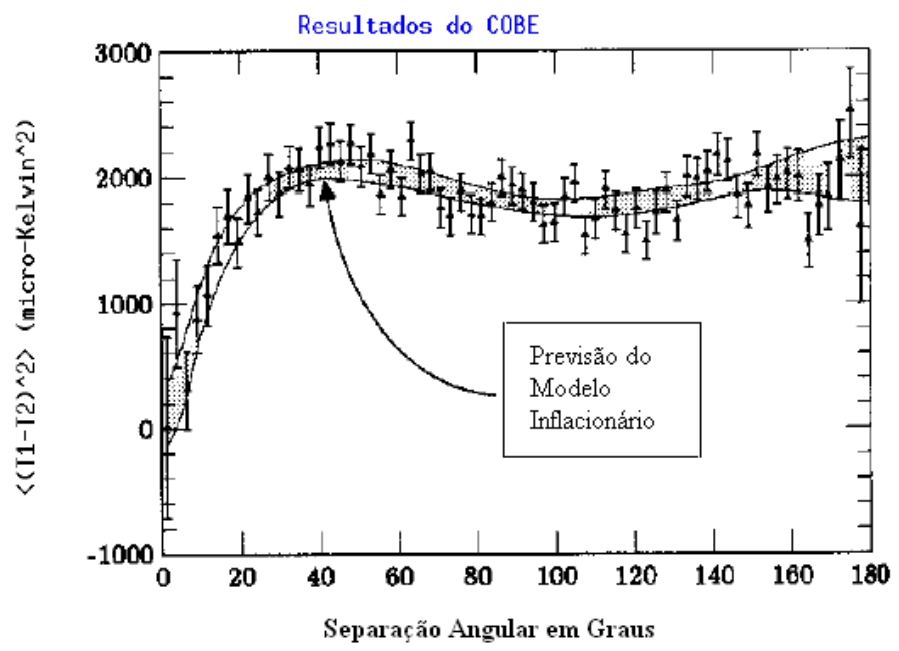

Fig. 5: Comparação das medidas de flutuação na temperatura da radiação do fundo do Universo obtidas pelo satélite COBE, com as previsões do modelo inflacionário. Os observadores do COBE mediram a diferença de temperatura entre duas regiões do céu, separadas por um certo ângulo, e calcularam o quadro dessa diferença: $\left(T_{1}-T_{2}\right)^{2}$, medida em microkelvins $\left(10^{-6}\right) \mathrm{K}$. Calculando-se a média dessa quantidade, para diferentes pares de direções, obtém-se uma medida estatisticamente significativa. Os modelos inflacionários podem calcular a forma desse espectro, mas não sua magnitude, de modo que a magnitude foi ajustada aos dados. Mas a forma, invariante de escala, isto é, que tem aproximadamente o mesmo valor para pequenas separações e grandes separações, é um dos maiores sucessos da teoria inflacionária, já que os modelos tradicionais do Big-Bang não têm qualquer forma de calcular esse espectro. 
A matéria escura não emite radiação eletromagnética e, portanto, somente podemos detectá-la através da força gravitacional que ela exerce sobre os objetos. A detecção da existência de matéria escura vem do estudo do movimento: movimento de estrelas individuais em galáxias, e o movimento de galáxias em aglomerados de galáxias. Quando aplicamos a lei da gravitação a esses movimentos, detectamos que a massa é muito maior que a massa visível em estrelas e gás.

O que é essa matéria escura? Se sua quantidade for de 5 a 10 vezes maior do que a de matéria luminosa, ela poderia se constituir de partículas normais (bárions); prótons e nêutrons, não condensados em estrelas, poeira ou gás, senão deveríamos detectá-los. Poderia, porém, ser composta de buracos negros (objetos colapsados gravitacionalmente), anãs marrons (objetos degenerados, mas de massa inferior a estrelas e maiores que Júpiter), e planetas (que não geram sua própria luz). As observações de microlentes gravitacionais na nossa Galáxia indicam que somente cerca de $2 \%$ da matéria está na forma de estrelas colapsadas. A matéria escura deve ser composta de partículas exóticas ainda não detectadas na Terra, como áxions, ou monopolos magnéticos.

\section{A idade do Universo}

Qual é a idade do Universo?

A matéria total do Universo gera atração gravitacional, em que objetos atraem outros objetos (inclusive a luz, pela relatividade geral). Assumindo-se que a constante cosmológica $(\Lambda)$ é nula, ou seja, que a energia do vácuo (repulsão) é nula, essa atração deve diminuir a expansão, o que implica que, no passado, a expansão era mais rápida. A idade do Universo pode ser calculada no limite superior, assumindo que a quantidade de matéria é pequena e que, portanto, não reduziu a velocidade de expansão significativamente. Podemos, então, estimular a idade máxima do Universo, $t_{o}$, calculando o tempo que as galáxias distantes, movendo-se à mesma velocidade de hoje, levaram para chegar aonde estão. Como a lei de Hubble, que relaciona a velocidade de expansão das galáxias, $v$, com a distância a esta, $d$, é dada por $v=H x d$, e $v=d / t_{o}$, então $t_{o}=1 / H$. Atualmente, o valor da constante de Hubble, $H$, está medido entre 70 e $78 \mathrm{~km} /(\mathrm{s} \mathrm{Mpc})$, resultando em $t_{o} \leq 12$ a 14 bilhões de anos $\left(1 \mathrm{Mpc}=\right.$ mega parsec $\left.=3,086 \times 10^{19} \mathrm{~km}\right)$. Levando-se em conta a possível desaceleração causada pela atração gravitacional, a idade é $t \geq \frac{2}{3} t_{o}$, isto é, entre 9 e 14 bilhões de anos. Por outro lado, calculando-se a idade das estrelas mais velhas conhecidas, as estrelas dos cúmulos globulares e as anãs brancas, obtêm-se 
entre 12 e 14 bilhões de anos. Mas se a constante cosmológica não for nula, o Universo está acelerando e sua idade é maior do que $H^{1}$.

Qual é a evolução química do Universo? O Universo se esfria enquanto se expande. Depois de 0,01 s do Big Bang, a temperatura do Universo era de $T=$ $10^{11} \mathrm{~K}$. Depois de 3 minutos, a temperatura já tinha baixado a um bilhão de graus Kelvin, ainda 70 vezes mais quente que o Sol. Depois de 380.000 anos, a temperatura se reduzira a meros $3000 \mathrm{~K}$. A uma temperatura de $T \geq 6 \times 10^{9} \mathrm{~K}(T \leq 1 \mathrm{~s})$, a colisão de 2 fótons pode gerar um par elétron-pósitron, por conversão de energia em massa $\left(E=m c^{2}\right)$. Para gerar prótons, a temperatura tem de ser maior que $10^{14} \mathrm{~K}$ $(\mathrm{t} \leq 1$ milissegundo). A época até uma idade de um milissegundo é chamada de era hadrônica, pois podia formar hádrons (prótons e nêutrons).

Note que, para tempo menor que $10^{-44} \mathrm{~s}\left(T \cong 10^{32} K\right)$, o chamado tempo de Planck [Max Karl Ernest Ludwig Planck (1858-1947)], as teorias físicas conhecidas não se aplicam mais, pelo princípio da incerteza: $\Delta E x \Delta t \geq \hbar\left(\hbar=1,05 \times 10^{-34} J s\right)$. No tempo de Planck, o raio do horizonte do Universo do Universo (raio visível) era $R_{U} \cong 10^{-33} \mathrm{~cm}$. O raio do Universo que contém toda a energia que se transformou na matéria hoje observada era menor que um centésimo de centímetro.

O raio do horizonte é derivado usando-se a relatividade geral, enquanto que o raio do Universo que contém que contém toda a energia é derivado usandose a mecânica quântica (princípio da incerteza de Heisenberg [Werner Karl Heisenberg (1901-1976)], e essas duas teorias não são compatíveis entre si. As teorias físicas se aplicam para tempos maiores que o tempo de Planck. No modelo patrão do Big Bang, logo após o tempo de Planck, o Universo estava em rápida expansão, com temperaturas colossais e altíssima densidade, uma situação lembrando muito uma explosão.

Gamow calculou a quantidade de deutério $(p+n)$ que se formaria nesse caso. Era possível obter-se a percentagem observada de deutério, muito maior do que poderia ser formado no interior das estrelas. Se essa matéria não estivesse banhada por uma radiação de certa intensidade, formar-se-ia muito mais deutério do que o observado.

Gamow, em 1948, previu que restos dessa radiação deveriam ainda estar banhando todos os corpos celestes. Tal radiação foi detectada, em 1964, como a radiação do fundo do Universo.

O deutério é um hidrogênio pesado, pois seu núcleo contém um próton e um nêutron. Embora observado no gás interestelar, no sistema solar e mesmo nos espectros de quasares, o deutério não pode ser formado nas estrelas. Quando uma 
estrela se forma por colapso de uma nuvem de gás interestelar, qualquer deutério nessa nuvem é destruído (convertido em hélio), mesmo antes da estrela se tornar quente o suficiente para iniciar a fusão do hidrogênio. Portanto, o deutério, como a maior parte do hélio, é um fóssil do Big Bang. Quando o Universo está esfriando, quanto maior o número de átomos em um volume no espaço (densidade), menor a quantidade do deutério que sobrevive, porque a maior parte se converte em hélio.

A teoria do Big Bang prevê que houve um pequeno excesso de matéria sobre a antimatéria (1 parte em 100 milhões), ou toda a massa seria aniquilada. Quando o Universo tinha $t=10^{-3} \mathrm{~s}$, sua temperatura era da ordem de $T \cong 10^{29} \mathrm{~K}$. A essa temperatura, a energia média por partícula é da ordem de $10^{16} \mathrm{GeV}(1 \mathrm{GeV}=$ 1 bilhão de elétron volts), a energia em que as teorias de Grande Unificação prevêem efeitos importantes, como a violação da conservação de número bariônico, e a possibilidade da formação de partículas super-massivas, o bóson de Higgs, predito por Peter Ware Higgs (1929-), em 1964. Essas partículas são instáveis, mas de longa vida, e podem, teoricamente, dar origem a esse pequeno excesso de matéria sobre a antimatéria.

Em 1964, James H. Christenson, James Watson Cronin (1931-), Val Logsdon Fitch (1923-) e René Turlay (1932-2002) conseguiram observar que, no decaimento da partícula neutra kaon, ou méson $\mathrm{K}$, existe uma pequena diferença $(0,2 \%)$ a favor da matéria, em relação à antimatéria produzida.

Cronin e Fitch receberam o prêmio Nobel em 1980 pela descoberta, demonstrando experimentalmente, que existe assimetria no Universo ${ }^{6}$.

Prótons e nêutrons começam a ficar ligados em núcleos quando o Universo tinha $3^{\mathrm{m}} 46^{\mathrm{s}}, T \cong 900$ milhões $\mathrm{K}$, formando deutério $(\mathrm{p}+\mathrm{n})$, e hélio $(2 \mathrm{p}+2 \mathrm{n})$, até uma idade de 4 minutos. O hélio formado é de, aproximadamente $25 \%$ em massa, próximo do observado.

Nesse modelo, após 4 minutos, a temperatura já é muito fria para formação de outros núcleos mais pesados. Depois de 380000 anos, $T \cong 3000 \mathrm{~K}$, os elétrons combinam com os núcleos, formando átomos neutros. Como não existem, então, mais elétrons livres para espalhar os fótons, o Universo passa de opaco para transparente e, a partir de então, a matéria e a radiação evoluem independentemente. Essa radiação de $3000 \mathrm{~K}$, viajando a uma velocidade de 2 milionésimos abaixo da velocidade da luz, é o que detectamos como radiação do fundo do Universo a

\footnotetext{
${ }^{6}$ Chen Ning Yang (1922-) e Tsung-Dao Lee (1926-) receberam o prêmio Nobel em 1957 por suas investigações da paridade.
} 
aproximadamente $3 \mathrm{~K}$. Somente milhões de anos depois as estrelas e as galáxias começam a se formar. Desde a formação das estrelas mais velhas, somente $10 \%$ da massa de hidrogênio inicial pode ter sido convertida em hélio, por fusão nuclear no centro das estrelas. A maior parte desse hélio ainda está no interior das estrelas. Portanto, a maior parte dos $25 \%$ de hélio observado no gás interestelar e na atmosfera das estrelas foi formada no Big Bang.

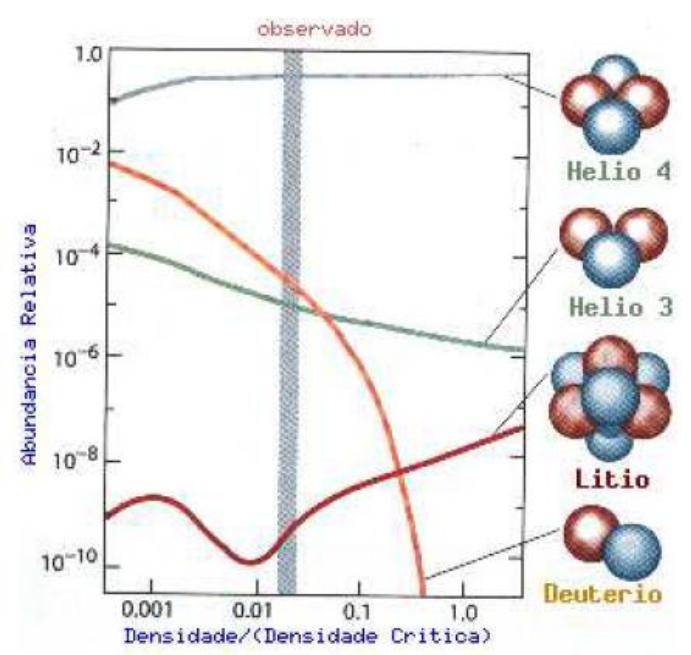

Fig. 6: Essa figura mostra como a abundância dos elementos formados depende da densidade de prótons e nêutrons, no modelo padrão de Big Bang, em termos da densidade crítica (densidade necessária para parar a expansão do Universo). Se o número de prótons e nêutrons for alto, mais freqüentemente eles colidem e mais hélio-4 é produzido. As abundâncias de deutério e hélio-3 decrescem quando aumenta a densidade porque núcleos são formados por uma seqüência de reações incompleta. Dado tempo suficiente, o deutério e o hélio-3 se transformam em hélio-4. Já o lítio-7 é produzido por várias reações e, portanto, depende da densidade de forma mais complexa. A nucleossíntese no Big Bang só formou os elementos leves: hidrogênio, deutério, hélio e lítio. Todos os elementos químicos mais pesados foram produzidos mais tarde, no interior das estrelas. 


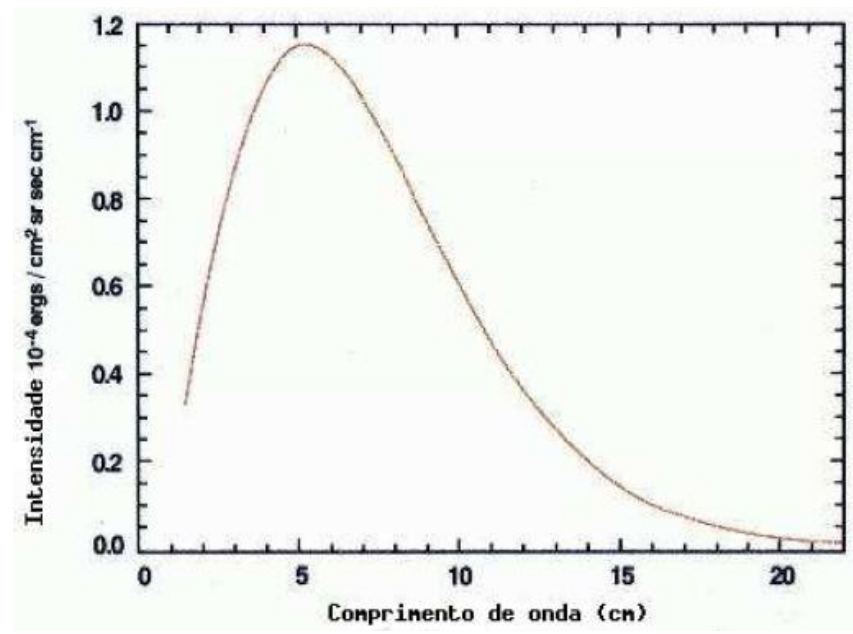

Fig. 7: Resultados do experimento FIRAS do satélite COBE, mostrando que a radiação do fundo do Universo segue mesmo a lei da radiação de Planck.

\section{COBE}

Em 18 de novembro de 1989, a NASA lançou um satélite chamado Cosmic Background Explorer (COBE), para analisar detalhadamente a radiação do fundo do Universo, operando na faixa de microondas.

Como planetas, estrelas, galáxias e nuvens de gás emitem muito pouco em microondas, o satélite pode enxergar diretamente a luz que o Universo emitiu quando passou de opaco para transparente, na chamada época da recombinação, cerca de 380 mil anos depois do Big Bang.

Os dados obtidos pelo COBE, mostrados na Fig. 6 e divulgados por John Cromwell Mather (1946-), cientista coordenador do projeto COBE, se ajustam perfeitamente a um corpo negro com temperatura de $2,726 \mathrm{~K}$, com uma incerteza menor que $0,01 \mathrm{~K}$.

Essa é a temperatura predita para a radiação do gás quente de quando o Universo se formou, visto com um desvio para o vermelho correspondente, pois a expansão do Universo estica o comprimento de onda pelo mesmo fator que o Universo se expande entre a emissão e a observação. 
Se o Big Bang tivesse sido caótico, por exemplo, o espectro observado não seria perfeitamente o de um corpo negro, mas seria distorcido para o azul, pelo decaimento das estruturas caóticas.

Cada metro cúbico do Universo contém, em média, 400 milhões de fótons e somente 0,1 átomos.

Em outro experimento do satélite COBE, divulgado em abril de 1992, por George Fitzgerald Smoot III (1945-), da Universidade da Califórnia em Berkeley, também foram detectados pequeníssimas variações da temperatura nessa radiação (seis partes por milhão).

Nos modelos de formação de galáxias, essas flutuações são necessárias para permitir que a matéria formada posteriormente se aglomerasse gravitacionalmente para formar estrelas e galáxias, distribuídas em grupos, bolhas, paredes e vazios, como observamos.

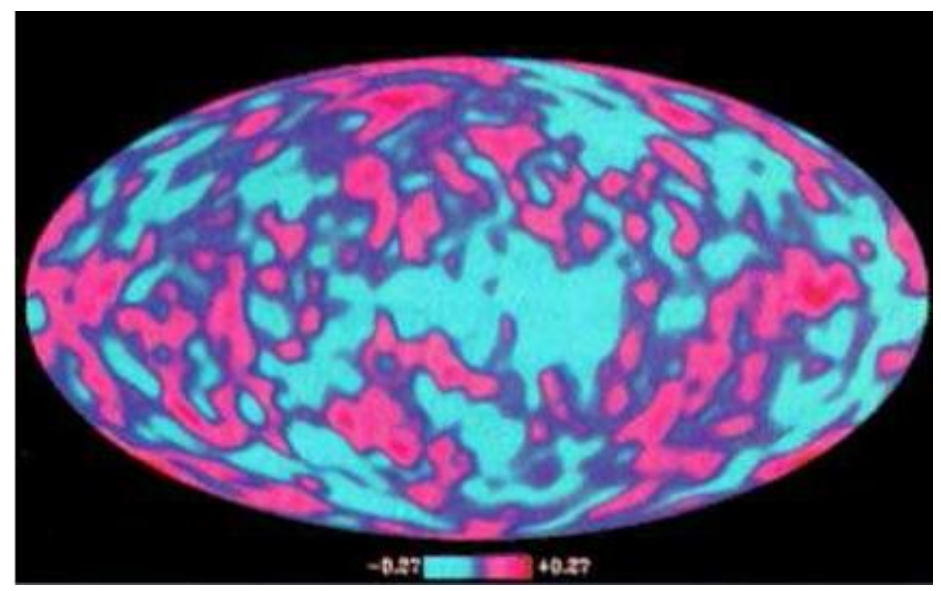

No modelo padrão, as estruturas do Universo são formadas a partir da amplificação gravitacional de pequenas perturbações na distribuição de massa inicial. Seria praticamente impossível haver a formação das estruturas observadas, como galáxias, estrelas, planetas e, portanto, da Terra e de nós mesmos, sem que houvesse variações de temperatura na radiação do fundo do Universo. Isso porque a radiação e a matéria estiveram em equilíbrio térmico no Universo primordial e, então, qualquer irregularidade na distribuição inicial de matéria seria refletida na distribuição angular dessa radiação. A detecção dessas flutuações até então era o principal ponto faltante na consistência da teoria do Big Bang e da formação e evolução do Universo. As flutuações de densidade observadas pelo COBE poderiam ser oriun- 
das de cordas cósmicas geradas nas transições de fase, ou poderiam ser simples flutuações normais de uma distribuição gaussiana de densidade.

Com o esfriamento do Universo, eventualmente a matéria se condensa em galáxias, estrelas se formam, evoluem e morrem, e elementos mais pesados, como carbono, oxigênio, silício e ferro vão gradualmente sendo sintetizados nas estrelas, e espalhadas no meio interestelar por explosões de supernovas. Esse gás é depois concentrado em outras estrelas, e em planetas e, possivelmente, em corpos de seres humanos, em alguns desses planetas!

O Universo tornou-se transparente quando a temperatura caiu para $T=3000 \mathrm{~K}$ e os elétrons se combinaram com os prótons, formando átomos de hidrogênio e hélio.

Esse evento chama-se época da recombinação, ou superfície de último espalhamento. Ele ocorre em deslocamento para o vermelho (redshift), $\mathrm{z}=1000$, já que a temperatura da radiação atualmente é de $3 \mathrm{~K}$, e

$$
z=\frac{T_{\text {inicial }}}{T_{\text {atual }}}=\frac{3.000 \mathrm{~K}}{3 \mathrm{~K}}=\frac{R}{R_{\text {atual }}}
$$

onde $\mathrm{R}$ é o raio do Universo.

A energia gravitacional de estrelas, galáxias e cúmulos de galáxias, dividida por $m c^{2}$, energia de repouso, corresponde a $10^{-5} \mathrm{e}$, portanto, a dinâmica desses objetos é não relativística. Essa também é a razão entre a temperatura média da radiação do fundo do Universo (Cosmic Microwave Background) e a temperatura das flutuações que deram origem às estrelas, galáxias e dos cúmulos de galáxias, já que representam o avermelhamento gravitacional (redução de energia) necessário para que os fotóns escapem do campo gravitacional.

A abundância observada de deutério, e também a de hélio, indicam que a densidade bariônica (de matéria normal) não pode ser maior do que 0,1 da densidade crítica. Entretanto, o movimento das galáxias em cúmulos de galáxias requer que a densidade total seja pelo menos 0,2 da densidade crítica. Grande parte da matéria escura precisa ser exótica. Portanto, sabemos que a densidade de matéria atualmente é próxima da densidade crítica, mas não existe evidência observacional de que a densidade total seja igual à densidade crítica. 


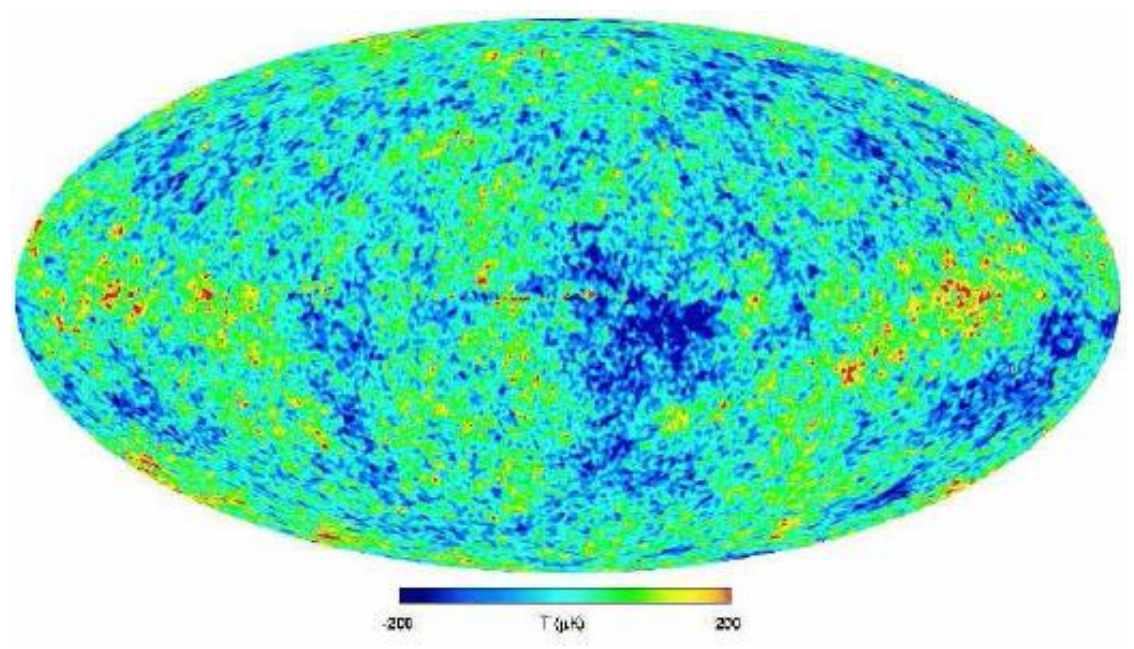

Fig. 8: Mapa do céu obtido pelo satélite Wilkinson Microwave Anisotropy Probe (WMAP) da NASA, lançado em 2001, com resolução angular de $0,22^{0} \mathrm{em}$ $93 \mathrm{GHz}$, divulgando por Charles L. Bennett (1956-) e colaboradores em 2003, 2008 e 2010. As regiões vermelhas são mais quentes (200 $\mu \mathrm{K}$, do que a média) e as azuis mais frias $(-200 \mu \mathrm{k})$. Os resultados após sete anos de dados, analisados por Gary F. Hinshaw (1961-), David Nathaniel Spergel (1961-), e colaboradores, indicam que a idade do Universo é de $(13,78 \pm 0,11)$ bilhões de anos (o primeiro pico no espectro de distribuição angular, em $263,8^{\circ} \pm 0,1^{\circ}$, é proporcional à distância à superfície de desacoplamento), que a matéria normal corresponde a $(4,61 \pm 0,15) \%$ da energia total (a amplitude do pico acústico é proporcional à densidade bariônica), $(23,2 \pm 0,13) \%$ de matéria escura e $(72,2 \pm 0,15) \%$ de energia escura (constante cosmológica) ou quintessência (energia com pressão negativa), completando a massa crítica prevista pelo modelo inflacionário ( $\left.\Omega=\frac{\rho_{\text {obsevado }}}{\rho_{\text {critico }}}=1,022 \pm 0,043\right)$. As observações indicam, ainda, que as primeiras estrelas se formaram (377 \pm 3 ) milhões de anos (dada pela detecção de reionização em $z=10,4 \pm 1,2)$ depois do Big Bang, o que indica que os neutrinos não dominam a evolução da estrutura, ou eles teriam dificultado a aglomeração do gás, retardando o nascimento das primeiras estrelas. A reionização pode ser detectada pela polarização causada pelo espalhamento dos fótons da radiação de fundo pelos elétrons livres ionizados pela formação estelar. 


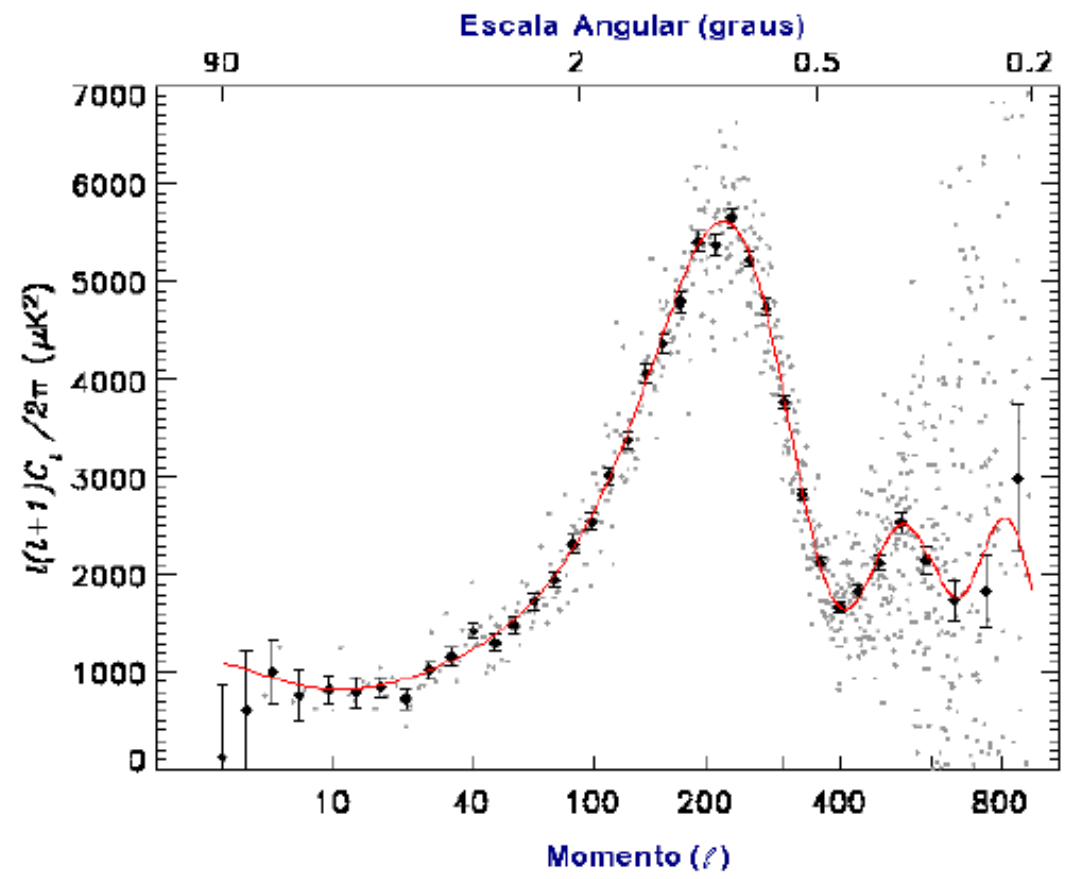

Fig. 9: Decomposição em esféricos harmônicos das flutuações observadas pelo WMAP. Se o Universo é aberto, as flutuações devem ser máximas em escaladas de $0,5^{\circ}$. Se $O$ Universo é plano, as flutuações devem ser máximas em escaladas de $1^{\circ}(\ell \cong 220)$. Se o Universo é fechado, as flutuações devem ser máximas em escalas maiores que $1^{\circ}$. A separação angular é dada por $\theta=\frac{180^{\circ}}{\ell}$. 